\title{
July 2018 Interim Report on the Progress of the MarXiv Repository
}

\section{General Information}

This document reports on the first ten months of operation of the MarXiv repository for the ocean and marine-climate sciences. OCTO (Open Communications for The Ocean) began work in earnest on the project in October 2017. This report is based on progress made through 30 July 2018.

We are very grateful to the David and Lucile Packard Foundation for their generous support of the MarXiv project. This report is a modified version of our first Interim Grant Report to the Packard Foundation. We hope this helps other projects learn from our experiences. Questions and comments may be directed to MarXiv's Project Manager, Nick Wehner, at nick@octogroup.org.

This report is made available under a CC-BY license. It may be cited as: OCTO (2018) July 2018 Interim Report on the Progress of the MarXiv Repository. 10.31230/osf.io/te9gv.

\section{Executive Summary}

As of 30 July 2018, there are 213 papers in MarXiv. Downloads from MarXiv since the start of the project have surpassed 9100. Adding up the downloads from MarXiv of otherwise pay-walled papers, we have saved the marine conservation field over $\$ 125,000$ in per-article purchase and rental fees. That figure is currently growing at over $\$ 1500$ per day. MarXiv is on pace to save the marine conservation field significantly more money than the project costs to operate.

Usage of MarXiv has greatly exceeded our expectations. From estimated Google Analytics Snapshots for the whole months of January through June 2018, we have had nearly 15,000 visitors to the MarXiv.org repository.

We have found that an individual email request asking an author to share a single, recently-published paper in MarXiv is the most effective way to grow the repository. After an author shares one paper, we send a thank-you email asking if they have other papers 
to share. Sometimes this will net us several (in one case 16) papers from a single author.

To make sending these personalized email requests scalable, we have built a "MarXiv Messaging System". The system generates individualized email requests to authors given the appropriate metadata from a paper (which we provide in a YAML file). The system then sends these emails using the bulk email delivery platform SparkPost. With SparkPost, we can track if messages are bounced, rejected, delivered, and/or opened, helping us to fine-tune our requests in ways that resonate with authors -- and don't get blocked by spam filters.

Our MarXiv Summaries have been popular with the authors of the articles summarized, particularly those for whom English is a second language. Our new monthly MarXiv Summaries newsletter is one of OCTO's most successful newsletters as measured by open rate. In addition, MarXiv's summarized research has been discussed regularly on OCTO's brand new podcast OCTOPOD, with over 400 plays to date, and will be featured on the popular Speak Up for Blue podcast starting Fall 2018.

The MarXiv Ambassadors have served as our "guinea pigs" in testing new tools and strategies. With their help, we've found that some publishers are much more receptive than others to sharing papers currently in the peer-review process (e.g., Don't bother asking Wiley if they take the SPARC Author Addendum -- they don't!)

Researchers from both the 2018 Ocean Sciences Meeting and the 2018 Salish Sea Ecosystem Conference expressed interest and excitement about MarXiv. We are hopeful future conferences will allow us to spread MarXiv's tools and opportunities even further.

Collaborations with our fellow preprint services on the OSF Preprints framework have helped MarXiv tailor its communications. In early August 2018, MarXiv personnel and other OSF Preprint services are discussing a joint advertising campaign to raise awareness of preprints among the general "science-interested" audience -- researchers and consumers alike.

\section{Activities, outputs, outcomes, and progress toward goals and objectives}

The key in our first year has been testing several different strategies with article authors to recruit their papers into MarXiv, then conducting rapid adaptive management in response.

We started our communications push by leveraging OCTO's multiple discussion lists, newsletters, and other communications assets (MPA News, MEAM, OpenChannels 
Weekly Update, OpenChannels Literature Update, MPA List, Community List, EBM Tools listserv, EBM Tools Discussion listserv, and more). Right off the bat, this yielded us a few star authors who shared their work in MarXiv. We have been able to use their papers -- and their tweets promoting their work -- in our communications ever since.

However, the volume of papers added in the first few months was, although steady, slow. Our mass communications didn't recruit as many papers as they attracted unfulfilled promises to share papers.

So we switched to direct one-on-one communications with authors instead. Our one-onone emails were individually written to authors about papers we would like to see in MarXiv. We tried several different styles of emails to find more effective ways of stating our message. We wanted data on these individualized requests -- which were fairly timeintensive to write -- to help us determine which templates resonated with authors more effectively.

\section{The MarXiv Messaging System (aka NickBot)}

So we created a MarXiv Messaging System to provide a database and several custom scripts to track papers that we'd like to add to MarXiv, generate initial request emails to authors, and send two follow-up email requests to those authors who haven't responded to earlier emails. We jokingly call this system internally "NickBot". With this system, we are no longer limited by how long it takes us to write emails, but in how long it takes us to find the papers we want and the relevant author contact information. We typically track about 20-30 paper requests a week now. We have also adapted our request templates to provide links to the publisher's exact self-archiving policies, give a sample preprint and postprint, and clarify more explicitly that we cannot host the Version of Record.

Based on our experience so far, each preliminary email we send to an author requesting a paper has a $10 \%$ chance of leading to a successful deposit. However, combining the original request with both a follow-up email after three weeks (to account for out-of-office away messages) and a second follow-up after another three weeks, we have a $28 \%$ success rate of getting the requested paper submitted to MarXiv -- a significant increase.

We have tested nearly a dozen iterations of our various email requests. The latest testing has shown that authors need to be explicitly told the self-archiving policies for their particular journal. Authors seem to rarely click through to read the policies themselves (we currently don't track clicks so we don't know for sure). As such, we are working to rebuild our MarXiv Messaging System from scratch to offer much more custom emails. In the meantime, we are sending three different templates: one for papers published by Elsevier, where there is no longer an embargo on postprints; one for papers published by everyone else, mentioning an embargo from six months to two years on the postprint and linking to the publisher's policies; and one for "Gray/Transient 
OA" papers (which are free for an unknown, limited time but not openly licensed) requesting authors to make these papers freely-available forever with MarXiv.

\section{Translation to non-English}

Because postprints often carry licensing restrictions from publishers (typically CC-BYNC-ND), these manuscripts are not fully open. One major limitation is that these works cannot be translated into other languages. English is hardly the universal language of climate and conservation sciences. So we feel strongly that individuals and NGOs should have the ability to translate research to reach new audiences. As such, we are now (as of early July) expressing this fact to authors. Since this change, nearly all submitted copies of papers published by Elsevier have been the unrestricted preprint. We are thrilled!

\section{Significant cost savings to the field}

Based on our email response rates to authors, touting the benefits to them from posting their papers in MarXiv (i.e., increased readership and citations) has not proven to be a motivating factor for them to share their work.

However, we have noticed that authors seem to be motivated by the cost savings MarXiv has provided the field: we have received numerous comments from authors about the costs of academic papers to those without institutional subsidies in response to our paper requests. As of July 30, downloads of pay-walled articles that are now available via Green Open Access in MarXiv would equate to $\$ 125,427.80$ saved in perarticle rental/purchase fees from the start of the project. And the savings are accelerating. As of July 16 this figure was growing at over $\$ 1000 /$ day, while as of July 25 it was growing at $\$ 1500 /$ day.

\section{Costs and benefits of guiding authors on-on-one}

We have found that most authors need to be guided one-on-one through nearly every step of the process: determining which papers they can share, which versions are under embargo, and the submission process itself. We have also found that oftentimes authors are too technology-averse to add required cover pages to papers (i.e., with required text from the publisher) or even to deposit the paper in MarXiv, so Nick has been doing much of this himself on behalf of authors.

Obviously there are costs to such hands-on, time-intensive guidance of authors. A benefit, however, is that leaving the deposition to Nick raises the quality of a paper's metadata: authors often forget to provide the link to the Version of Record's DOI, which prevents the MarXiv version from being linked to the record in Google Scholar. Other times they only list themselves as the sole author, despite there being multiple authors of the paper. Since Nick can only edit papers he is explicitly listed as an administrator for 
by the author, it is more efficient if Nick does the deposit (it's all muscle memory for him at this point!). However, we remain hopeful that authors will become comfortable filling out web-forms in time.

\section{Authors' frustration with adding co-authors}

Some authors have expressed frustration with the OSF Preprints deposition process: namely, the lack of an intuitive $\mathrm{UI}$ and the time-consuming process of adding co-authors (which requires searching for the co-author -- and if they don't exist in the OSF database, manually adding the author's name and email address for each one). The coauthor process takes time, especially for papers with dozens of co-authors and/or when email addresses for co-authors are unknown. The Center for Open Science (COS) is working on a new version of its Preprints API which we hope to utilize to offer direct submissions to the MarXiv repository from our documentation portal at MarXivinfo.org. With this, we could (hopefully) iron out these kinks. The biggest benefit this native functionality would provide internally is automatically making Nick an administrator on shared preprints -- he cannot edit any paper in MarXiv that he is not explicitly made an admin for by the author. So with our own deposition utility, we could ensure Nick becomes an admin automatically for all papers submitted via MarXivInfo.org (but not on the repository itself, MarXiv.org). Regrettably, COS does not plan on allowing preprint admins the ability to edit papers in their repositories by default.

\section{MarXiv Ambassadorships}

We advertised the MarXiv Ambassadorships far and wide, but only received six completed applications for ten spots during the first year. To help these limited Ambassadors make a larger impact, we reallocated the remaining budget for the year to these first-year Ambassadors to make promotional materials: stickers, notecards, and other handouts. These have been popular. We quickly ran out of our supply of 1500 MarXiv stickers and notecards.

Unfortunately, the onsite trainings that MarXiv Ambassadors have conducted thus far (representing half of the current Ambassadors) have been attended by very few scientists. One training at Simon Fraser University had only one person in attendance, while a second training had three. A training by the OCTO Team at the University of Washington was attended by just 10 students (and zero faculty). As such, we have discontinued further in-person trainings by OCTO for the time being.

While some of the Ambassadors have been helpful spreading our messages on social media, the impact from the Ambassadors overall appears to be limited. We suggest scrapping the plan for ten Ambassadors for Year 2. Instead, we propose re-allocating those funds to an advertising budget. Nick is currently discussing with our fellow preprint leaders (SocArXiv, InarXiv, PaleorXiv, etc.) if we could pool resources to create a joint "Community Preprint Services" advertising campaign to amplify our impact through 
favorable print, podcast, and other outlets. Early discussions show many existing preprint services would like to join us in this endeavor. We will chat more on a call in early August. Rather than granting stipends, we would instead solicit an unlimited number of unpaid MarXiv Ambassadors, similar to what BioarXiv does with its ambassadors.

\section{Training sessions}

The planned weekly Zoom-meeting virtual trainings on how to use MarXiv were of limited success. The attendees were almost exclusively MarXiv Ambassadors and Advisory Board members. After a few weeks of zero attendance, the weekly trainings were cancelled. Instead, now, researchers and academic departments can request trainings at will. Nick regularly uses the system to help authors one-on-one rather than in a large group, which has helped researchers feel more comfortable since they can ask questions immediately, rather than being lectured to in a webinar-style training.

\section{MarXiv Summaries}

MarXiv Summaries have been helpful in spreading word of the project. We integrate new summaries into our weekly OpenChannels Literature Update newsletter, typically sharing two Summaries each week to the readership of roughly 2000 individuals. In addition we created a new MarXiv Summaries newsletter, sent the first week of each month, which shares all the Summaries we have written the previous month (8-12 Summaries). This newsletter reaches 6000 readers. Both newsletters have a $30 \%$ open rate, higher than the conservation industry standard.

The Summaries are read by researchers and practitioners alike. Researchers have been supportive of our efforts to translate their work to a manager audience -- especially those researchers for whom English is not their native language. NGO researchers and activists, managers, and government employees have all expressed gratitude on getting the gist of research explained succinctly and without jargon. While the number of downloads of Summaries from MarXiv has been somewhat small (average download count is 36), we know from clicks on the Newsletters that our audience tends to simply read the PDF in the preview window on MarXiv, likely because the summaries are short. Downloads of the full-text of summarized research almost always spike after a Summary is shared.

We have heard from several scientists how much they appreciate MarXiv Summaries for helping to increase the impact of their work. The Scientific Watch newsletter of the Mediterranean Protected Areas Network (MedPAN) has included several MarXiv Summaries and other papers shared in MarXiv, too. We may have even catalyzed a new research collaboration on using drones to monitor beach litter thanks to sharing a MarXiv Summary on this topic through our MarineDebris. Info listserv. A scientist at the University of Haifa wrote that he was looking into research on the topic when he saw our 
summary, and was thrilled to know he could potentially collaborate with others for a grant proposal he was writing.

\section{A tale of three audiences}

Currently MarXiv is a tale of three audiences:

1. Practitioners and students (particularly outside the US and UK) who increasingly depend on MarXiv to access and understand new and emerging science

2. Researchers who understand what MarXiv is doing and the value of open access literature to those on the front lines of marine conservation science and climate science

3. Researchers who are wary of angering academic publishers and/or fear they may be violating copyright law by sharing their work with MarXiv, despite evidence to the contrary

The first two audiences are good. The third presents a challenge.

It has become clear from our testing of email templates that the majority of academic authors contacted by MarXiv either don't believe what MarXiv is explaining about preprint rights, do not read our emails at all, or are not comprehending our emails. We track opens (though not clicks) on the emails sent through the MarXiv Messaging System. From this data, we can see that nearly every author we email opens at least one of the requests we send (we send up to three per author). But we hear back from only $\sim 15 \%$ of the total authors for each email we send.

Of that $15 \%$, many of the replies simply send us the full text of the paper, which in most cases is illegal for us to post. Others send us the "author's link" for free access for a limited time, which we have no way to share in MarXiv. And other authors reply with questions or confusion that would be cleared up if they read our original email more closely.

Despite these setbacks, with enough persistence and one-on-one education and support, we are able to share almost a third of the papers we ask for.

\section{Hypothesis annotation framework}

As of 20 July 2018, MarXiv now has integrated support with the Hypothesis annotation framework. We hope that by promoting this functionality to the field, we may be able to encourage researchers to share their manuscripts in MarXiv before publication in established journals. Of the 213 papers in MarXiv, only four are pre-acceptance preprints (papers submitted to MarXiv before acceptance in a traditional journal). Hopefully with the community peer review that Hypothesis helps to create, researchers 
will see more value in sharing their work in MarXiv before academic publishers put barriers on sharing.

\section{Indicators}

1. Number of preprints added to the MarXiv repository

a. Grant goal was 1000 preprints by end of Year 1; 2500 preprints by end of Year 2.

b. As of 30 July 2018, we had a total of 213 papers in MarXiv:

i. $\quad 37 \%$ are papers we have directly solicited from authors via email requests or asking over social media.

1. 78 journal articles, 1 thesis

ii. $35 \%$ are reports we've shared from NGOs, research conducted by OCTO staffers, or MarXiv Summaries. We anticipate this proportion to decline over time as more authors share their papers directly.

1. 34 Summaries, 35 reports/other docs, 5 works by OCTO staffers

iii. $\quad 28 \%$ are unsolicited papers that were added to MarXiv by the authors themselves, or where the author subsequently shared additional papers in MarXiv beyond the one we requested directly.

1. 48 journal articles, 4 unpublished preprints, 4 reports, 2 theses, 1 blog, 1 conference paper

c. As of 30 July 2018, there have been 9113 downloads from MarXiv, equating to $\$ 125,427.80$ in cost-savings to the marine conservation community by avoiding per-article download fees on papers that were previously only available from behind a publisher paywall.

d. We will not meet our target of 1000 papers by the end of Year 1, and it appears unlikely we will meet our target of 2500 by the end of Year 2 .

i. Figure: Number of papers in MarXiv, showing projected growth trends. The lower trend line indicates our initial strategy of sending personalized email solicitations to each author. The steeper trend line indicates our shift to programmatic emails in late March 2018. 


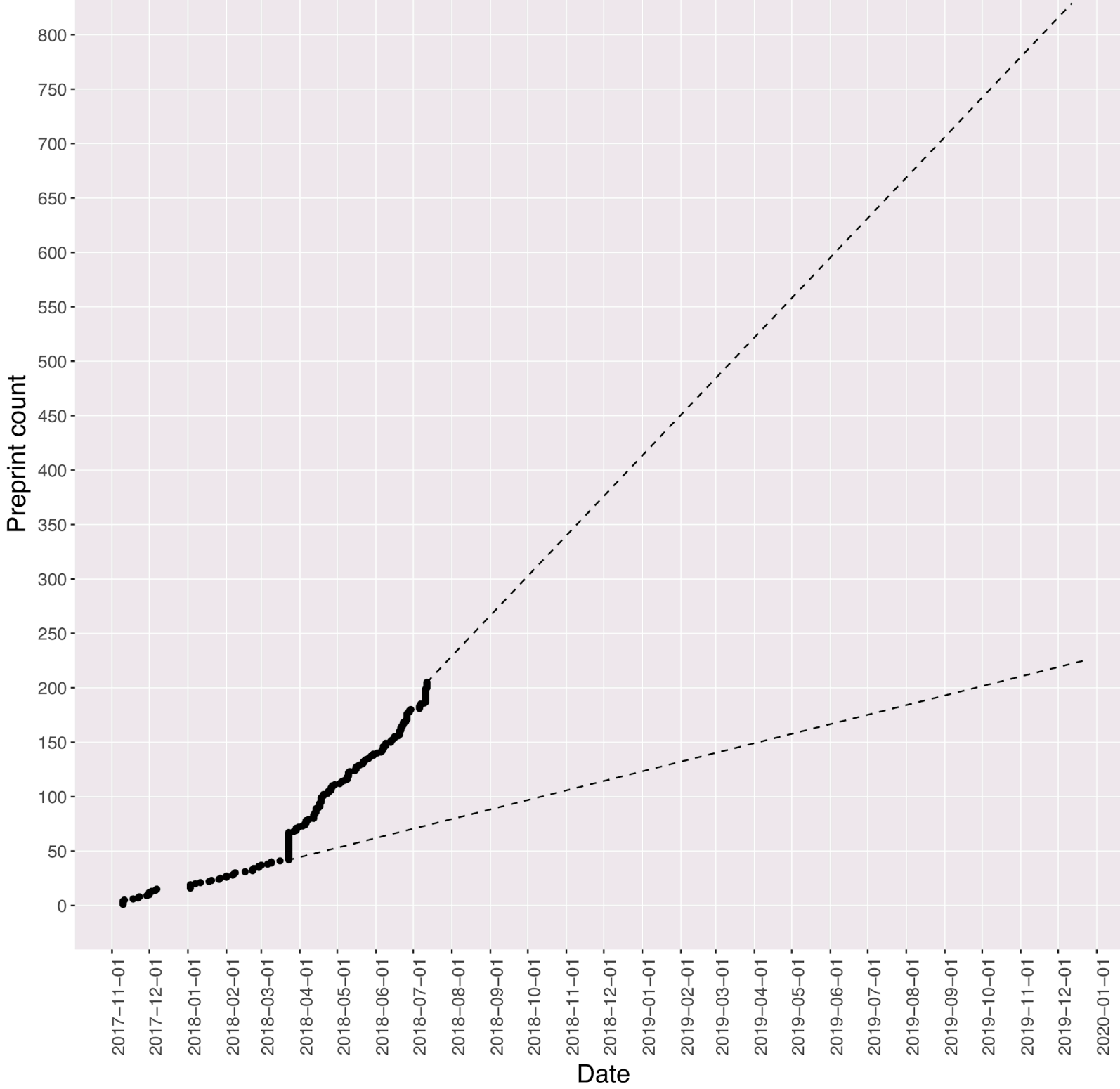

ii. Based on the projections above, our shift from individual email requests manually written by OCTO staffers to our databasecentric programmatic approach has had a significant positive impact on growth. At our current growth rates, we anticipate we'll reach approximately 350 papers by the end of Year 1, and approximately 750 papers by the end of Year 2 .

iii. We believe that further refining our email requests to authors -and the follow-ups we send when we don't hear back -- will increase our growth rates before the end of Year 1. In addition, network effects should increase the number of unsolicited papers shared by authors as more of the marine conservation science 
and marine climate science communities become aware of MarXiv.

2. Annual number of unique users and unique pageviews for the MarXiv platform.

a. Goal was 2000 unique users and 4000 unique pageviews by the end of Year 1; 5000 unique users and 8000 unique pageviews by end of Year 2.

b. We requested Google Analytics data for MarXiv.org from the Center for Open Science in early July, but we have yet to receive this data. Monthly stats are automatically emailed to us in Google Analytics Snapshots for this domain, so we can approximate our cumulative traffic from these. Snapshots are available for the months of November 2017, and January through June 2018.

i. Cumulatively (excluding the missing month of December 2017 and the most recent data for July 2018), MarXiv.org has seen 14,797 visitors.

c. The MarXiv documentation website, MarXivInfo.org, has seen 1,554 users across 2,086 sessions since it went live in early December 2017 through 25 July 2018. The site has served 3,997 pages with 3,198 of those unique pageviews.

d. Usage of both websites has thus greatly exceeded our expectations! But we caution that the data on MarXiv.org is just an approximation at this time. We can see from the usage of the repository, demand for freelyavailable science is much higher than we had expected.

3. Awareness of MarXiv in the marine conservation science and marine climate science communities, and in the marine practitioner and policymaker communities.

a. In accordance with the grant proposal, this will be accomplished at the midway point of Year 2.

b. Anecdotally, with all the individual email requests to authors that we're sending and the distribution/readership of the Summaries, we think we're well on our way to building awareness in these respective communities. Though, to be clear, we haven't done a robust survey at this time.

4. Annual number of summaries of top-level research.

a. Goal was 100 summaries by the end of Year 1; 200 by the end of Year 2 .

b. As of 30 July 2018, we had 34 published summaries. We expect to produce another 24 by the end of Year 1, placing us at around 58 summaries by the end of Year 1. Regrettably, this means we'll be under the target number of MarXiv Summaries by about 40\% for Year 1. 
c. A number of factors have contributed to the lower than anticipated number of MarXiv Summaries:

i. Primary among them is the slow start in getting high-impact papers shared in MarXiv at the start of the project. As a result, we had very few papers to summarize for the first few months of Year 1.

ii. A handful of Summaries are still awaiting edits and approval from the lead authors, which we do not anticipate ever receiving. Because of this, we're now giving authors a week to provide comments/edits. When we don't hear back, we publish the Summary as is.

iii. Finally, we do not wish to summarize low-quality papers, as we believe doing so could cause more harm than good for the field. Despite many of the papers in MarXiv being published in respected, peer-reviewed journals (namely Marine Policy and Ocean \& Coastal Management) we have noted questionable statistical methods being used routinely, making the conclusions of the papers less than fully reliable. Authors also sometimes over-sell the outcomes of their own experiments in the discussion and conclusion sections. In these cases, we avoid summarizing the papers so as not to promote flawed research within our communities.

\section{External Events}

\section{Elsevier allows sharing of postprints without embargo period}

Happily, and unexpectedly, Elsevier's Director of Scholarly Communications, William Gunn, told us via Twitter to follow the same rules Elsevier set for arXiv and RePEc: namely, authors can share their postprints in MarXiv without the standard 2-year embargo period.

This is backed by Elsevier's official policy, which librarian Josh Bolick pointed out to us (also via Twitter), that allows authors to subvert the embargo altogether by simply sharing the postprint on their own personal websites or blogs prior to submission to MarXiv. Specifically, Elsevier mandates that postprints be shared with a CC-BY-NC-ND license. Authors are permitted to share postprints on their own personal websites or blogs immediately. Thanks to the mandated Creative Commons license, anyone may share that copy for non-commercial purposes.

This policy change has helped us immensely! The authors we contact over email are often wary of sharing their pre-peer-reviewed manuscripts for fear of typos and other errors being seen, or in rare cases when peer-review has substantially changed the 
document. The waived embargo period for Elsevier has accounted for the majority of recent submissions to MarXiv.

\section{Some countries do not allow Federal employees to copyright works}

In both the US and Canada, works produced by employees of the Federal government are not eligible for copyright. Specifically in the US, as long as one author of an academic paper is employed by the Feds (not a contractor working for the Feds, though, from what we can tell) and that paper is part of the author's regular duties, that work cannot be copyrighted (17 U.S. Code $\S 105)$. Thus, these authors do not have a copyright to transfer to a publisher as part of the Copyright Transfer Agreement (CTA) process. Despite this very short and clear Federal law, many Federal agencies seem to ignore it. We've found several papers where at least one author is a NOAA employee, yet the papers are copyrighted and pay-walled by the major publishers (mostly by Elsevier). In these situations, we've been sharing the final "Version of Record" in MarXiv which has helped us add more papers to MarXiv without waiting for an embargo.

Regrettably the authors of these papers have thus far ignored our requests to properly publish the papers without copyright. We have tried to find the right people to talk to in NOAA, without success so far. We only found out about this thanks to a chat with a USGS employee at the Salish Sea Ecosystem Conference earlier this year. USGS spends significant time convincing publishers to abide by the law. Evidently, NOAA does not follow USGS's lead. We are still actively working to find ways to rectify this problem, which seems widespread in NOAA and BOEM, at the very least.

We have submitted FOIA requests to NOAA, EPA, and BOEM seeking lists of papers published by agency staffers since the law took place, and delineating those that are unlawfully copyrighted. We have also requested what action the agencies are taking to ensure compliance with the law. We are hopeful the requests will at least help us get the agencies to notice there is a problem in the first place.

\section{Lessons Learned}

From a communications standpoint, we have found that researchers react poorly to any implication that they're part of the problem of scientific access. References to "The Ivory Tower" in our initial communications, for examples, elicited pushback and prickliness from researchers, rather than acknowledgement. We have learned not to focus on the bad aspects but rather to communicate the positives of greater access to science.

We have learned that some researchers are likely never to be convinced that Open Access/Open Science are good. We have had researchers tell us publicly on Twitter that they only want to share the "pretty version". Others have told us that universities and 
NGOs need to cough up the money for journal subscription fees and just stop complaining about the costs. We have had researchers accuse us of trying to get them sued/arrested by encouraging them to share papers in MarXiv. We just continue to make our case, and test new ways of making it.

Resistance extends to some researchers' perceived time availability. We regularly have researchers send us emails explaining how they don't have time to share a paper, or even to send it to us to share on their behalf. To test this, we have timed how long it takes for untrained individuals to find a paper on a computer, get a proper version to share in MarXiv, and do the uploading process. It takes three minutes on average. Which is likely significantly less than the time needed for the resistant researchers to write their emails to us.

Because of these pervasive inefficiencies, academics predominantly need introductorylevel guidance when it comes to explaining self-archiving and Green Open Access. No matter the evidence we provide, many researchers are just not going to read our messages or click through to resources that back-up our claims. They need to be handheld each step of the way. Older/more senior academics will usually punt our requests to their post-docs or assistants. We believe the best hope for sparking change is by educating new and emerging researchers about the benefits of self-archiving, as this group generally seems interested in learning from us.

We originally expected that researchers would be motivated by the many studies showing how Green Open Access can benefit them personally: increased citations, larger readership, community support. But these have not proven to be motivating factors. Strangely, what has seemed to work best is simply persistence. Researchers seem to get tired of us asking them repeatedly (yet politely) for papers, or they get tired of coming up with excuses, so we eventually get the paper if we keep asking what's holding them back. Some researchers also seem motivated by the cost-savings MarXiv has provided to the field -- currently over $\$ 125,000$ in per-article rental/purchase fees avoided on Green OA papers.

The economics of the academic world do not help the situation: many of the most senior scientists in our field are editors of high-profile, pay-walled journals. They have a direct financial incentive to maintain the status quo. To some extent MarXiv represents a threat to their business model, and thus their paychecks. We have tried partnering with scholarly societies, but many of these groups rely on subscriptions to pay-walled journals for funding. MarXiv again poses a threat to these groups, so we have yet to establish any official partnerships. Others in our situation may find they first need a critical mass of supporters with voting power in these groups to spark change.

We do know that light public shaming works, but we use it sparingly so as not to end up on the bad side of the research community. Pointing out on social media, for example, 
that a paper about how restricted science hurts conservation in fact costs $\$ 50$ to read will get those authors to make the paper Open Access one way or the other.

Most importantly, we have learned that lack of knowledge about the academic publishing process is rampant -- even among the editors of journals themselves! In some cases we have had to inform journal editors what their own policies are. Researchers largely have no idea what the copyright restrictions are on their work. We have encountered some who believe that since they wrote it they can share it anywhere they want, even if they transferred their copyright away. You should start all conversations about self-archiving with the absolute basics, because the "facts" that academics think they know are usually incorrect.

On the upside, we have been sending handwritten thank-you notes to authors (including some of the promotional materials for MarXiv, like stickers and notecards explaining how MarXiv fits into the publishing workflow) in cases where we can find their physical mailing address online. We've had some of those authors write us back saying it's the first handwritten thank-you note they have received since they were children. Simple things like that can make you good friends in the fight for Open Science!

Regularly chatting with our preprint partners at the Center for Open Science has helped us learn what's been working for those groups, too. We wouldn't have made stickers if it didn't help out the other groups. And a few of the other preprint groups are now making informational notecards like ours. Perhaps most importantly, our preprint partners have helped keep us level-headed despite the challenges! Turns out they all face similar challenges as we do. 